\title{
Deserción escolar en las madres adolescentes en el contexto fronterizo Norte de Santander y Táchira*
}

\author{
María-Antonia Cuberos ${ }^{1}$; Marisela Vivas-García; Neida Albornoz-Arias; \\ Rina Mazuera-Arias ${ }^{4}$; Myriam-Teresa Carreño-Paredes ${ }^{5}$
}

* Capítulo de libro producto de los proyectos de investigación: 1) Análisis comparativo de la Maternidad adolescente en la frontera Norte de Santander - Táchira. Fecha de inicio: enero de 2017. Fecha de finalización: diciembre de 2017. Universidad Simón Bolívar - sede Cúcuta - Colombia. 2) El Táchira en Cifras 2014. Maternidad y embarazo en adolescentes. Universidad Católica del Táchira, Venezuela. Fecha de inicio: 01 de enero de 2014. Fecha de finalización: diciembre de 2017.

1 Licenciada en Educación, Universidad Católica Andrés Bello, Venezuela. Ingeniero de Sistemas, Universidad Nacional Abierta, Venezuela. Doctor en Ciencias Gerenciales, Universidad Nacional Experimental Politécnica de la Fuerza Armada, Venezuela. Especialista en Sistemas de Información. Universidad Católica Andrés Bello, Caracas. Docente e investigadora, miembro del Grupo Altos Estudios de Frontera (ALEF) de la Universidad Simón Bolívar Cúcuta, Colombia Docente investigadora de la Universidad Católica del Táchira, San Cristóbal, Venezuela.

Correo: m.cuberos@unisimonbolivar.edu.co

2 Licenciada en Educación mención Ciencias biológicas. Universidad Católica Andrés Bello, Venezuela. Doctor en Innovaciones Educativas, Universidad Nacional Experimental Politécnica de la Fuerza Armada, Venezuela. Magíster en la Enseñanza de las Ciencias Básicas, Universidad Nacional Experimental del Táchira, Venezuela. Especialista en Evaluación Educacional, Universidad Valle del Momboy, Venezuela. Docente e investigadora miembro del Grupo Altos Estudios de Frontera (ALEF) de la Universidad Simón Bolívar, Cúcuta, Colombia Docente Asistente de la Universidad Católica del Táchira, San Cristóbal, Venezuela.

Correo:m.vivas@unisimonbolivar.edu.co

3 Contador Público Universidad Católica del Táchira, Venezuela. Doctora en Ciencias Sociales y Jurídicas, Universidad de Córdoba, España. Especialista en Análisis Bursátil y Gestión de Carteras, IEB Madrid, España. Universidad Simón Bolívar, Facultad de Administración y Negocios, Cúcuta, Colombia. Miembro del Grupo de investigación Altos Estudios de Frontera (ALEF), Universidad Simón Bolívar, Colombia. Docente e investigadora de la Universidad Católica del Táchira, San Cristóbal, Venezuela.

Correo: n.albornoz@unisimonbolivar.edu.co; albornoz@ucat.edu.ve

4 Abogada Universidad Católica del Táchira, Venezuela. Doctora en Derecho en el programa Derecho de Familia y de la Persona Universidad de Zaragoza, España. Especialista en Derecho Administrativo Universidad Católica del Táchira y Derecho Tributario. Universidad Santa María, Venezuela. Universidad Simón Bolívar, Facultad de Ciencias Jurídicas y Sociales, Cúcuta, Colombia y Docente e investigadora en la Universidad Católica del Táchira, San Cristóbal, Venezuela.

Correo: r.mazuera@unisimonbolivar.edu.co; rmazuera@ucat.edu.ve

5 Licenciada en Educación, Universidad Francisco de Paula Santander, Cúcuta. Psicóloga en formación, Universidad Nacional Abierta y a Distantica (UNAD). Especialista en Orientación Educativa y Desarrollo Humano, Universidad del Bosque, Bogotá. Universidad Simón Bolívar, Facultad de Administración y Negocios, Cúcuta, Colombia. Miembro del Grupo de investigación Altos Estudios de Frontera - ALEF, Universidad Simón Bolívar, Colombia

Correo: m.carreno@unisimonbolivar.edu.co 


\title{
RESUMEN
}

El capítulo presenta una descripción de lo que acontece con la deserción escolar en las madres adolescentes en un contexto geográfico fronterizo colombovenezolano, en específico en el estado Táchira y el departamento Norte de Santander. Se aborda la deserción escolar como un fenómeno en donde intervienen múltiples factores que se integran haciéndolo complejo. El desarrollo del capítulo se hizo, a partir de los datos tomados de los resultados de las investigaciones: "El Táchira en cifras, 2014, maternidad y embarazo en adolescentes" realizada por la Universidad Católica del Táchira, Venezuela y "Maternidad de las adolescentes en Norte de Santander" adelantada por la Universidad Simón Bolívar sede Cúcuta, Colombia. En ambas se empleó la metodología cuantitativa, con enfoque transversal y diseño de campo, recolectando datos de madres adolescentes mediante dos encuestas estructuradas aplicadas una en cada ámbito. El análisis de los datos se realizó mediante el empleo de la estadística descriptiva; se llegó a los resultados que indican la existencia de varios factores intervinientes en la deserción escolar de las madres adolescentes estudiadas, bien sea anteriores al embarazo o posteriores a este.

Palabras clave: deserción escolar, madres adolescentes, Norte de Santander, Táchira.

\section{SCHOOL DROPOUT RATES AMONG ADOLESCENT MOTHERS IN THE NORTHERN BORDER OF SANTANDER AND TÁCHIRA}

\begin{abstract}
The chapter presents a description of what happens to school dropout in adolescent mothers in a Venezuelan Colombo border context, specifically in the Táchira State and the Norte de Santander Department. School dropout is addressed as a phenomenon in which multiple factors intervene that are integrated making it complex. In the development of the chapter, the quantitative methodology was followed, based on the data taken from the results of the researches: "Táchira in figures, 2014, maternity and pregnancy in adolescents" by the Universidad Católi-
\end{abstract}


ca of Táchira-Venezuela and "Maternity of the adolescents in Norte de Santander" advanced by the Simón Bolívar University Cúcuta-Colombia; in both, the quantitative methodology, with transversal approach and field design, was used, collecting data of adolescent mothers through two structured surveys applied one in each area. Data analysis was performed using descriptive statistics, results were obtained indicating the existence of several factors involved in the school dropout of the adolescent mothers studied, either before or after pregnancy

Keywords: school dropout, pregnancy and teenage motherhood, Norte de Santander, Táchira

\section{INTRODUCCIÓN}

El vocablo deserción proviene del latín desertio. La Real Academia (2017), define deserción como abandonar las obligaciones o los ideales. De allí que al relacionar deserción con escuela, se determina el abandono de los compromisos escolares o los ideales que el estudiante puede tener con respecto a su formación.

Para Espinoza, Castillo, González, Loyola y Santa Cruz (2014), es el abandono progresivo de la escuela como espacio cotidiano, que ocurre como un proceso, configurado con muchos y variados eventos que puede ser estudiado desde distintos ámbitos sin considerar si es forzado o voluntario. Se dice que surge como respuesta a una situación social como la pobreza, ante la desigualdad económica profunda vinculada con la distribución de la riqueza y de los recursos simbólicos y culturales asociados (Estrada, 2014; Troncoso, 2008).

La deserción escolar según otros autores, como Aguilar y Viniegra (1999), acontece ante la violencia, abuso y maltrato a menores por integrantes del sistema escolar, repercutiendo sus consecuencias en diversos ámbitos de la vida personal y social al crearse una violencia simbólica (Bourdieu y Passeron, 2002), que generalmente conduce a la apatía hacia el estudio de 
los jóvenes y de sus padres para que continúen los estudios; así como a comportamientos de mala conducta y problemas vinculados con la edad.

También se ha planteado como causas de la deserción la no presencia de planteles educativos, su accesibilidad y escasez de profesores, o problemas familiares que son concernientes con la ejecución de labores en el hogar, el embarazo y la maternidad (Espindola y León, 2002; y Piña y Pontón, 1997). Al respecto, Mazuera y Arias (2017) dicen que el embarazo adolescente no es la causa principal del abandono escolar; sin embargo, tiene peso en el abandono temporal o definitivo en los estudios. Goicovic (2002) y Azevedo, et al. (2013) expresan que la deserción escolar en el caso de las adolescentes antes y después del embarazo se motiva en la pertenencia a familias disfuncionales, que no ofrecen apoyo para que continúen sus estudios, así como hogares pobres o, también porque tienen menos aspiraciones y metas por alcanzar.

En este sentido, se planteó investigar acerca de la asociación existente entre la deserción escolar y la maternidad en adolescentes, o su embarazo, entendido este como aquel que se presenta en el tiempo transcurrido desde la menarquia, y/o cuando la adolescente es aún dependiente de su núcleo familiar de origen (Organización Mundial de la Salud-OMS, 2009).

La maternidad adolescente representa según autores un problema de alta complejidad en el campo biomédico con consecuencias socioculturales y psicológicos que se ha transformado en una preocupación para diversos ámbitos de la sociedad (Molina, et al., 2004; Issler, 2001; Fernández, Carro, Oses y Pérez, 2004). Sin embargo, existe otra posición con respecto a la maternidad en adolescentes. Pacheco (2016) plantea que cuando el contexto está caracterizado por la pobreza, la maternidad adolescente puede llegar a convertirse en la forma de lograr mejoras en las condiciones de vida de la joven, pues ante las circunstancias que viven, proceden a construir proyectos de vida con su pareja y la maternidad, transformando 
en beneficio su experiencia de embarazo; de allí que bien podrían abandonar la escuela para dedicarse a la crianza y al hogar.

En América Latina se han venido presentando tasas de deserción escolar tempranas muy elevadas, que se convierten en un factor propicio para la creación de desigualdad de oportunidades de una generación a la siguiente, afectando así las posibilidades futuras de bienestar, pues la educación es fundamental para la inclusión social de las personas al ser un derecho humano básico y un medio que capacita a los sujetos para que ejerciten sus derechos (Espíndola y León, 2002). De allí que se convierten en desafíos: impedir el abandono de la escuela antes de terminar el ciclo básico y reducir la deserción en el ciclo medio si se busca alcanzar los Objetivos de Desarrollo sostenible (Organización de las Naciones Unidas, 2015).

Abordar las estrategias conducentes para cumplir los retos, implica el conocimiento de las características, debilidades, relaciones con otros fenómenos que en torno a la deserción se presentan en los diversos contextos de Latinoamérica; de este modo, se estaría adelantando un camino para construir la agenda de las políticas educativas. La presente investigación tiene como propósito la comparación del fenómeno de la deserción escolar desde la maternidad adolescente en el estado Táchira y Norte de Santander, pretendiendo generar conocimiento que contribuya a la construcción de las estrategias y acciones componentes de las agendas públicas en educación orientadas al alcance de oportunidades de bienestar y desarrollo.

Al plantear un contexto de estudio a objeto de observar el fenómeno deserción escolar-embarazo y maternidad en adolescentes, se consideró la zona de frontera colombovenezolana, en específico en el departamento Norte de Santander (Colombia) y en el estado Táchira (Venezuela). Se buscó responder a cuáles son los factores que intervienen en la deserción escolar de las madres adolescentes del contexto de estudio, partiendo de dos inves- 
tigaciones realizadas: "El Táchira en cifras, 2014, maternidad y embarazo en adolescentes" ejecutada por la Universidad Católica del Táchira, Venezuela y "Maternidad de las adolescentes en Norte de Santander" adelantada por la Universidad Simón Bolívar sede Cúcuta, Colombia.

\section{DESERCIÓN ESCOLAR Y MATERNIDAD ADOLESCENTE}

Lo que significa la deserción escolar. Es el alejamiento y abandono paulatino de la escuela que experimenta el estudiante, (Espinoza et al., 2014), en un proceso que para Troncoso (2008), está configurado con muchos y variados eventos, afectando a la persona, a la familia, a la comunidad, a la escuela y a la sociedad a través de componentes espirituales, económicos, sociales, culturales, de valores y otros, pudiéndose abordar desde distintos ámbitos sin importar si ese proceso es forzado o voluntario. Sin embargo, puede aparecer como una expresión del prejuicio, de las preconcepciones que tienen sobre determinados alumnos, algunos actores claves existentes en el proceso educativo, como por ejemplo aquellas representaciones sociales que relacionan a las mujeres con roles originarios subordinados a los indígenas con una capacidad intelectual y de trabajo menor a los grupos de poder.

López, Marín y García (2012) sostienen que el estudiante, decide abandonar de manera voluntaria o forzada sus estudios motivado por la influencia negativa o positiva de situaciones internas o externas. La Universidad Nacional de Colombia (2010) plantea que la deserción escolar -al significar abandono-implica una toma de decisión crucial y dramática, que conduce a cambiar el curso de una vida, por ende, obedece a patrones interrelacionados que se han ido reproduciendo y concretando como formas de exclusión e invisibilidad social, pues el suceso o acontecimiento en la vida del estudiante que desencadena el abandono, lo margina socialmente.

Estrada (2014) dice que la deserción se corresponde con un acto individual de abandono como respuesta a una situación social; el sujeto deja 
la escuela al no poseer una meta clara y en específico una pertenencia a la institución o a un grupo de compañeros. Asimismo, Troncoso (2008) relaciona la deserción con la pérdida de lazos o espacios de sociabilidad ya que la escuela además de ser el sitio de aprendizaje formal también es un lugar de sociabilidad y aprendizaje informal, es decir, es la prolongación más natural de la socialización iniciada en la familia y el barrio.

En la misma perspectiva Vargas y Valadez (2016) y Tinto (2005) sostienen que al no existir integración social y académica en la institución educativa, las metas académicas y compromisos con la escuela de los estudiantes pueden modificarse, afectándose su permanencia; de este modo los autores dan importancia al tipo de escuela, los recursos, infraestructura, arreglos estructurales y composición de sus miembros, pues, todo en su conjunto posee la capacidad de conducir al desarrollo de ciertos climas escolares favorecedores a la integración en la institución y por ende, influenciar la continuidad escolar. Se denota así el valor que poseen las características institucionales como los estándares académicos, el sentido de pertenencia tanto de estudiantes como profesores a la institución educativa y los valores y creencias de los actores educativos en cuanto a la permanencia en la escuela.

Lo anterior significa que la integración juega un papel importante, pues, la vida académica representaría un factor para la reducción del abandono escolar, al convertirse aquella en una fórmula de apego o identidad institucional junto a los contenidos académicos y las relaciones de amistad y compañerismo entre los estudiantes. En la escuela se construye identidad con los demás al ser un espacio cotidiano; cuando ocurre la deserción se pierde la sociabilidad y sentido de vida, en virtud de que la ocurrencia de interrelaciones facilita el aprendizaje y la construcción de presentes y futuros, así se comparte el proyecto de vida y hasta se compara. Al existir alejamiento, la posibilidad del proyecto se interrumpe o se desvanece en el día a día (Troncoso, 2008).

\section{1}


Por consiguiente, la deserción escolar involucra autoexclusión si se sigue el planteamiento de Estrada (2014). Ahora bien, esa exclusión implica una imposición de ser y estar en el mundo adulto tal y como lo expresa Visu (2006 citado por Troncoso 2008), no estar, ser excluido, es una imposición que se presenta sin que existan las competencias adecuadas tanto en lo personal, afines a actitudes y valores, como competencias funcionales unidas al cumplimiento de tareas o funciones específicas y competencias técnicas o de conocimiento que se corresponden con el avance intelectual de los individuos, competencias que no solo se enlazan al mundo del trabajo sino además al mundo social y personal de las personas.

Sin embargo, Espinoza et al. (2014) asocian a la deserción no solo con exclusión sino también con la pobreza, como también lo plantea Troncoso (2008), para quien la deserción emerge de la desigualdad económica profunda que se relaciona con la distribución de la riqueza y de los recursos simbólicos y culturales asociados.

Para la Corporación Colombia Digital (2012), la deserción o abandono escolar, representa la separación del alumno del sistema educativo, renunciando a la escuela sin lograr un certificado académico. La encuesta nacional en el año 2010, realizada por la Corporación, hizo ver que tanto la deserción como la permanencia en la escuela son fenómenos multidimensionales, pues dependen del contexto social regional que incluye las condiciones de los planteles (distancia, infraestructura, discontinuidad en oferta de cursos, escaso uso de Tecnologías de Información y Comunicación (TIC)), de las condiciones familiares (problemas económicos, trabajo infantil, nivel educativo de los padres) y a condiciones individuales (ingreso tardío a la educación, pérdida de cursos, bajas expectativas de formación postsecundaria). Woods (2002) también plantea la existencia de factores que llama de riesgo y que se asocian con la escuela, los mismos estudiantes, la familia y la comunidad; el autor expresa que hay mayor probabilidad de que un estudiante abandone la escuela si se presenta una integración de estos factores.

\section{2}


En la investigación de Abril, Román, Cubillas y Moreno (2008) en Sonora, México, al aplicar una encuesta a 147 jóvenes sobre situación familiar, historia escolar, motivos de deserción y planes futuros, entre otros, hallaron que el $86 \%$ desertó de la escolaridad entre el primer y tercer semestre, con un promedio de calificación, en el último semestre cursado, de 7,49. Entre las causas reveladas para abandonar la escuela resaltaron las económicas, reprobación de asignaturas y falta de interés; además se encontró que el $93 \%$ de los encuestados no sentía satisfacción con el nivel de estudios alcanzado, no obstante entre sus planes no se halló el retomar tales actividades. En el caso de las mujeres, el estudio concluyó que son los factores económicos motivo de la deserción, y la reprobación de materias en el de los hombres. De este modo, se plantea una diferencia con otros estudios que expresan que mujeres abandonan la escuela, principalmente por embarazo.

Asimismo Estrada (2014), al estudiar la deserción escolar en la educación media superior en México, en específico en el estado de Sonora, obtuvo resultados sobre los principales factores que intervienen en la deserción juvenil: uno de ellos es el económico, pues, el joven tiene que trabajar, a ello se adiciona la deficiente formación en la educación básica que conduce a una exigencia mayor en la escuela media superior lo cual lleva a la tendencia de la reprobación del estudiante, convirtiéndose esto en el detonante (INNE, 2011 citado en Estrada, 2014), además, señala el autor que es en los subsistemas de educación media superior en donde se ofrece una formación bivalente y orientada hacia la incorporación al mercado laboral en donde se presenta tal característica.

Por otra parte, otros estudios como el de Piña y Pontón (1997) y Espindola y León (2002), plantean no solo el factor económico ante la escasez de recursos en el hogar que posibiliten enfrentar los gastos para que los jóvenes asistan a la escuela, o la necesidad de trabajar o buscar empleo. También se consideran factores de disponibilidad de planteles educativos a ese nivel, su accesibilidad e insuficiencia de profesores. Además, los

\section{3}


problemas familiares que atañen a niñas y adolescentes vinculados con la ejecución de labores en el hogar, el embarazo y la maternidad.

Para Aguilar y Viniegra (1999), la deserción escolar posee motivaciones en la violencia, abuso y maltrato a menores por integrantes del sistema escolar, trascendiendo sus efectos en diversos ámbitos de la vida personal y social. Además plantean los autores, que el problema de la deserción puede entenderse como resultado de ciertas condiciones respaldadas por la organización en el sistema educativo y que se ha ido convirtiendo en arbitrariedad cultural en la disposición del currículo escolar, al existir imposición de saberes propios afines con intereses dominantes, enlazados a las ideologías estatales en la escuela, medios de comunicación, entre otros, de un modo tal, que se expresa con la violencia simbólica (Bourdieu y Passeron, 2002).

Igualmente se suman a los factores anteriores, la falta de interés, la apatía hacia el estudio por parte, de no solo los jóvenes, sino también de sus padres para que prosigan los estudios. Otro factor ligado a la deserción se refiere al comportamiento del joven, lo que se llama mala conducta y problemas que se asocian con la edad (Espinoza et al., 2014; Espíndola y León, 2002). En las mujeres, dice Goicovic (2002), está también el embarazo y la falta de apoyo familiar para continuar estudiando, aunque para Azevedo et al. (2013), la deserción escolar de las adolescentes antes y después del embarazo se debe a la pertenencia a familias disfuncionales, hogares pobres y/o porque sus expectativas en cuanto a metas y aspiraciones son pocas.

¿Qué trae consigo la deserción escolar? Como efectos para los jóvenes desertores, se consideran los planteamientos de Goicovic (2002), Henao, González y Vargas (2007) y Weller (2007), sobre la imposibilidad de integrarse en función de los cambios que va imponiendo la sociedad al mundo laboral por la ausencia de preparación, lo cual impide la salida de la pobreza y la movilidad social, pues no existen oportunidades para 
insertarse en el mercado laboral $u$ obtener un empleo de calidad, si como mínimo no posee 12 años de escolaridad y es egresado de educación media superior; por ende, se ve impedido de mejorar sus condiciones de vida y su situación de pobreza.

En virtud de ello, se inicia un espacio de exclusión que puede conducir a riesgos con respecto a su integridad física y emocional, además de empobrecer el capital cultural que luego pasará a sus hijos, reproduciéndose la desigualdad educativa y social de una generación a otra que obstaculizan oportunidades de bienestar y con mayor énfasis en los sectores más pobres. A ello se une la problemática que se genera para el país, pues se afecta la posibilidad de aprovechar económicamente la estructura de edad a través del incremento de la capacidad productiva y financiera de los jóvenes (Weller, 2007).

Por consiguiente, la deserción escolar involucra costos sociales derivados de la presencia de una fuerza laboral con pocas competencias y por tanto difícil de calificar al no poseer niveles mínimos de educación; a lo que se añade la baja productividad del trabajo que, a su vez, implica menor crecimiento de las economías, pérdida de impuestos; asimismo el gasto requerido para el financiamiento de programas sociales y de transferencias a aquellos sectores que no generan recursos propios constituyen un costo social, uniéndose además, la reproducción intergeneracional de las desigualdades sociales, de la pobreza y su impacto sobre la integración y cohesión social que impide fortalecer y profundizar la democracia (Woods, 2002).

Lo que significa la maternidad adolescente. Según la Organización Mundial de la Salud (OMS, 2009) el embarazo adolescente sucede en el tiempo transcurrido desde la menarquia o primer período menstrual, y/o cuando la adolescente es aún dependiente de su núcleo familiar de origen. La menarquia aparece en la joven entre los 10 y 16 años, y la edad media considerada son los 12 años y medio; si bien varía entre países al considerar el nivel socioeconómico, teniendo influencia además, los factores hereditarios (Méndez, 2011).

\section{5}


El embarazo en la adolescencia es producto de un conjunto de factores que se integran en una perspectiva ecológica en consideración a la situación socioeconómica de la joven, los derechos que posee sobre su cuerpo, su decisión propia para embarazarse, su sexualidad y fecundidad, la cultura de su familia y su pareja.

La maternidad adolescente es uno de los problemas más complejos existente en la reproducción humana desde el punto de vista biomédico; posee un elevado riesgo de complicaciones durante el embarazo y parto por lo cual puede incrementar las tasas de morbilidad y mortalidad materna, perinatal y neonatal. A ello se unen efectos socioculturales y psicológicos con elevado costo personal, educacional, familiar y social (Molina et al., 2004). Para Issler (2001) este fenómeno se ha transformado en una preocupación muy seria para varios sectores sociales, en especial en la salud, en cuanto a la mayor ocurrencia de resultados desfavorables o por las implicaciones del aborto; así como en lo psicosocial, por los efectos adversos que tiene sobre la adolescente y sus familiares. Asimismo, el embarazo en la adolescencia es un problema que se relaciona con los resultados perinatales y por las implicaciones psicosociales que la maternidad trae apareada a esta edad y que tiene efectos en la joven madre sobre el cuidado y atención que pueda darle a su hijo, además de sus posibilidades de desarrollo personal (Fernández et al., 2004).

Considerando los planteamientos de Hopkins, Lambrou, Morse y Wallach (2005), se presentan en el embarazo adolescente mayores riesgos médicos como el síndrome hipertensivo, anemia, desnutrición, parto prematuro, bajo peso al nacer, mayor ocurrencia de muerte materna y perinatal, entre otros. Además efectos psicológicos en la madre como la depresión post-parto, baja autoestima, cambios en el proyecto de vida a lo que se unen desventajas sociales entre las que se encuentran bajos logros educacionales, deserción escolar, desempleo, mayor frecuencia de maltrato y abandono infantil, entre otros. 
Por otra parte, Pacheco (2016) plantea que el embarazo en la adolescencia es el resultado de unas circunstancias de desigualdad socioeconómica que llevan consigo pobreza, exclusión y vulnerabilidad, y si esta se encuentra muy pronunciada, las adolescentes elaboran proyectos de vida dentro de la unión y la maternidad, llegando a ser el embarazo una experiencia feliz y una solución beneficiosa. Al no poseer planes para el estudio, trabajo o realización personal, la adolescente no visibiliza el futuro, solo existe en ella el presente y así la maternidad es un elemento que le permite organizar su trayectoria de vida; aunque se presenten emociones como el miedo, soledad, inseguridad, vergüenza y desconcierto, logra afirmar su propia identidad, reconocimiento social y aproximarse al mundo adulto.

En la investigación sobre maternidad y embarazo en adolescentes realizada por Magallanes (2015) para el Observatorio Social de la Universidad Católica del Táchira (UCAT) se detectó que existió una recuperación de la pobreza por NBI para las mujeres que fueron primíparas para todos los subgrupos considerados en el estudio, al comparar por períodos cuatrienales y en el año en el cual se efectuó la investigación. De este modo, según los resultados se llega a considerar que la maternidad adolescente favorece el proyecto de vida, en especial para aquellas adolescentes de ambientes más empobrecidos; lo que converge con lo planteado por Pacheco (2016) para hábitats caracterizadas por la pobreza, la maternidad adolescente no es necesariamente una calamidad ya que con el esfuerzo van logrando cierta estabilidad mejorando sus condiciones de vida.

Ahora bien, Fuenmayor, Sulbarán y Bargarza (2014a) plantean que en América Latina la probabilidad de que las madres adolescentes sean pobres es siete veces más que en aquellas de mayor edad; mientras más pobre es el país, el nivel de procreación precoz es más elevado: al considerar solo partos de jóvenes entre 15 y 19 años, se encontró que el $15 \%$ del total de 13 millones de partos registrados en América Latina y el Caribe, recaen en

\section{7}


adolescentes. Se podría revisar la recuperación de la pobreza por NBI para esta área geográfica para corroborar o no el estudio realizado por Magallanes (2015) y los planteamientos de Pacheco (2016).

Los motivos que conducen al embarazo, sea un problema para la adolescente, o una forma de consolidar proyectos de su vida, según Pacheco (2016), se refieren a la búsqueda del reconocimiento que como adultas, la maternidad les concede, pudiendo escapar de conflictos familiares, como en el caso de aquellas niñas en estado de pobreza que cuidan hermanos menores, o que son responsables al asumir cargas del hogar con escaso reconocimiento social. Fuenmayor, Sulbarán y Bargarza (2014b), expresan que entre las razones del embarazo adolescente se encuentran el poco conocimiento de los adolescentes con respecto al tema, las debilidades en la educación de la sexualidad en la familia, lo que implica la escasa existencia de un trabajo educativo organizado y sistemático que la Organización Mundial de la Salud (OMS, 2009) vincula con la exigua preparación del claustro de profesores en las instituciones educativas para tratar esta temática.

¿Qué trae consigo la maternidad adolescente? Para la OMS (2009), al igual que para Williamson (2013), las consecuencias del embarazo en la adolescencia, bien sea inmediatas o duraderas, no solo están relacionadas con la salud, al estar en riesgo la vida de la madre y del recién nacido o generar un círculo de enfermedad; además son de índole social y económica, las cuales incorporan a sus familias al inducir la pobreza ya que si la madre adolescente deja de estudiar, no tendrá las aptitudes y oportunidades propicias para desempeñarse en un trabajo; así, se genera un costo económico para el país, al perderse los ingresos anuales que una mujer joven obtendría en su existencia si no hubiese sido protagonista de un embarazo precoz; de allí que altera el curso de toda su vida, el embarazo plantea un cambio radical, muy raras veces para bien. Por consiguiente, la maternidad 
adolescente, al considerarse un problema, trae consecuencias negativas en lo social y económico para las madres, las familias y la sociedad (Mazuera y Arias, 2017).

Del mismo modo, el Fondo de Población de las Naciones Unidas (UNFPA, 2014) en su boletín informativo, plantea que un embarazo en la adolescencia lleva consigo futuros truncados, limitaciones en las posibilidades de estudio, retiros o deserción del sistema educativo con lo cual la joven adolescente no llega a ser profesional para desempeñarse en un trabajo, por ende, la problemática del embarazo en la adolescencia además de un problema de salud, lo es también de desarrollo.

\section{La maternidad en la adolescencia y la deserción escolar en un contexto} geográfico fronterizo. Según datos estadísticos del Departamento Administrativo Nacional de Estadísticas DANE (2016), los nacimientos en Colombia ocurridos en ese año por edad de la madre representan el 0,84\% en el grupo comprendido entre los 10 y 14 años y el $20 \%$ de madres entre los 15 y 19 años; de los mismos, el 0,02 \% y el 0,63\% respectivamente corresponden al departamento Norte de Santander. En este, son los municipios de Cúcuta y Ocaña en donde se presentan más nacimientos para ambos grupos etáreos.

Con los resultados de la Encuesta Nacional de Deserción (ENDE) realizada en el año 2010 en Colombia, se dio a conocer, en el encuentro regional del año 2011, que la tasa de deserción en el país fue del 6,7 \%; en la socialización de resultados de la citada encuesta, se indicó que en Colombia la tasa de deserción para el 2009 fue del 5,15\%, sin embargo, en Norte de Santander fue de 5,65 \% (República de Colombia, 2011). Según la Corporación Colombia Digital (2012), la encuesta indicó además que según los directivos en un $24 \%$, uno de los factores causantes de la deserción es la maternidad o paternidad temprana. 
Con respecto a Venezuela, según el Fondo de las Naciones Unidas para la Infancia (UNICEF, 2017a) para el año 2014 entre los 15 y 19 años, la fecundidad adolescente por cada mil mujeres se había incrementado con respecto al 2011 en 3,61 \%, siendo para el 2014, el porcentaje de madres adolescentes de $23,4 \%$. Con respecto a la tasa de deserción en educación media por cada 100 entre el 2011 y 2012 fue del 7,8 \% (UNICEF, 2017b).

Ahora bien, considerando los dos contextos: ¿cuáles factores intervienen en la deserción escolar de las madres adolescentes? Para dar respuesta se emplearon datos provenientes de la situación actual de estudio, la actividad principal antes del primer embarazo, el abandono escolar antes y después del primer embarazo, motivos del abandono escolar, rendimiento escolar de las adolescentes antes de su embarazo o actual y con quien vive actualmente la adolescente.

\section{METODOLOGÍA EMPLEADA}

A objeto de dar respuesta a la interrogante planteada en este capítulo, se siguió la metodología cuantitativa, con un enfoque analítico univariado, se empleó estadística descriptiva (Hernández, Fernández y Baptista, 2006; Fierro, 2010). Se utilizó el método deductivo, pues se partió de la idea de que en la deserción escolar intervienen múltiples factores; por razón de ello, se partió de lo general, se indagó sobre la actividad principal antes del primer embarazo de la situación de cada adolescente en cuanto a estudio al momento de ser encuestada, sobre su rendimiento escolar antes de su embarazo o para el momento de la investigación, se pudo fijar la situación del abandono escolar antes y después del embarazo, los motivos del abandono y su situación acerca de con quién vivía al momento de la aplicación de la encuesta. 
Las variables consideradas se estudiaron por separado; se empleó la distribución de frecuencias para una tabla univariada, considerando términos porcentuales y en algunas veces se hace referencia al número de casos, así se cuantificó la realidad estudiada de cada ámbito (Táchira y Norte de Santander). Los datos que han servido de insumo para este capítulo se tomaron de los resultantes de las encuestas estructuradas (Hernández et al., 2006) utilizadas en las investigaciones de Magallanes, et al., (2015), realizada por la Universidad Católica el Táchira,Venezuela y Mazuera, et al., (2017), ejecutada por la Universidad Simón Bolívar sede Cúcuta, Colombia. En ambas, se siguió la metodología cuantitativa, con enfoque transversal y diseño de campo.

En el estado Táchira se consideró un espacio muestral de 1.963 casos de madres adolescentes con cuatro meses o más de embarazo para mayo de 2014 en los 29 municipios; la muestra empleada fue de 235 adolescentes en el cuarto o posterior mes de embarazo. En el departamento Norte de Santander, se consideró una población de 6.435 madres adolescentes, con edades entre los 10 y 19 años, con hijos menores de 18 meses, y agrupadas en tres cohortes (10-14, 15-17, 18-19), nacidos entre octubre de 2013 y marzo de 2015 en los 40 municipios de Norte de Santander; la muestra se determinó con afijación proporcional por municipio de residencia de la madre; se obtuvo un tamaño 406 madres adolescentes.

\section{RESULTADOS Y DISCUSIÓN}

\section{Resultados del estudio realizado en el estado Táchira}

Situación actual de estudio. En la investigación realizada en el estado Táchira, como se aprecia en la Tabla 1, existe un desequilibrio en cuanto a la situación de estudio, ya que muy cerca de los dos tercios del total de adolescentes encuestadas no estudian. 
Tabla 1

Situación actual de estudio

\begin{tabular}{c|c|c}
\hline Actualmente estudia & Frecuencia & Porcentaje \\
\hline Sí & 75 & 31,8 \\
No & 160 & 68,2 \\
Total & 235 & 100,0 \\
\hline
\end{tabular}

Fuente: Elaboración propia. Datos obtenidos de una muestra de 235 adolescentes encuestadas en el Estado Táchira en el proyecto: El Táchira en Cifras 2014. Maternidad y embarazo en adolescentes (período de ejecución enero de 2014 - diciembre de 2017), en la Universidad Católica del Táchira, Venezuela.

La actividad principal antes del primer embarazo. Sin embargo, según las respuestas dadas por las adolescentes encuestadas, cerca del $15 \%$ de ellas, antes del primer embarazo, no realizaban actividad escolar (Tabla 2). Se desprende que existió deserción escolar en las madres adolescentes objeto de estudio.

Tabla 2

Actividad principal antes del primer embarazo

\begin{tabular}{c|c|c}
\hline Actividad principal & Frecuencia & Porcentaje \\
\hline Estudiar y trabajar & 54 & 23,1 \\
Solo estudiar & 145 & 61,5 \\
Solo trabajar & 22 & 9,4 \\
Ni estudiar ni trabajar & 14 & 6,0 \\
Total & 235 & 100,0 \\
\hline
\end{tabular}

Fuente: Elaboración propia. Datos obtenidos de una muestra de 235 adolescentes encuestadas en el Estado Táchira en el proyecto: El Táchira en Cifras 2014. Maternidad y embarazo en adolescentes (periodo de ejecución enero de 2014 - diciembre de 2017), en la Universidad Católica del Táchira, Venezuela.

El abandono escolar antes y después del primer embarazo. Como se aprecia en la Tabla 3, según los datos recolectados, de las 160 adolescentes que dijeron no estudiar, aproximadamente dos tercios de ellas dejó de hacerlo antes del primer embarazo y más de un tercio dejó de estudiar al quedar embarazada. De ello se deriva que aunque existió alto abandono escolar, la motivación no fue precisamente el embarazo. 


\section{Tabla 3}

Abandono escolar antes y después del primer embarazo.

\begin{tabular}{c|c|c}
\hline Abandono escolar & Frecuencia & $\begin{array}{c}\text { Porcentaje } \\
\text { válido }\end{array}$ \\
\hline Antes del primer embarazo & 99 & 61,6 \\
Despues del primer embarazo & 61 & 38,4 \\
\hline
\end{tabular}

Fuente: Elaboración propia. Datos obtenidos de una muestra de 235 adolescentes encuestadas en el Estado Táchira en el proyecto: El Táchira en Cifras 2014. Maternidad y embarazo en adolescentes (periodo de ejecución enero de 2014 - diciembre de 2017), en la Universidad Católica del Táchira, Venezuela.

Motivos del abandono escolar. En la Tabla 4 se evidencia las razones por la que 99 adolescentes dejaron de estudiar antes del primer embarazo, las razones que tuvieron: no quiso estudiar más, no hizo falta $(34,8 \%)$, el haber culminado estudios $(27,5 \%)$, o por lejanía de la escuela, motivos económicos u otros $(37,6 \%)$. Se denota que las motivaciones se refieren a factores intrínsecos como el no querer estudiar, circunstanciales como haber ya concluido sus estudios, económicos y otros.

\section{Tabla 4}

Motivos del abandono escolar

\begin{tabular}{|c|c|c|}
\hline Motivos del abandono escolar & Frecuencia & $\begin{array}{c}\text { Porcentaje } \\
\text { válido }\end{array}$ \\
\hline Terminaste los estudios & 27 & 27,5 \\
\hline $\begin{array}{c}\text { Escuela o liceo muy lejos de casa } \\
\text { No quise estudiar más / No hace } \\
\text { falta } \\
\begin{array}{c}\text { Por el costo (útiles, transporte, } \\
\text { gastos) }\end{array}\end{array}$ & 6 & 5,8 \\
Otro motivo & 7 & 34,8 \\
Total & 24 & 7,2 \\
\hline
\end{tabular}

Fuente: Elaboración propia. Datos obtenidos de una muestra de 235 adolescentes encuestadas en el Estado Táchira en el proyecto: El Táchira en Cifras 2014. Maternidad y embarazo en adolescentes (periodo de ejecución enero de 2014 - diciembre de 2017), en la Universidad Católica del Táchira, Venezuela.

\section{3}




\section{Rendimiento escolar de las adolescentes antes de su embarazo o actual.}

En la Tabla 5 se muestra que antes de quedar embarazadas o en el momento de ser encuestadas, menos de la mitad de las adolescentes manifestó que era bajo o muy bajo, en contraposición al resto de madres adolescentes que dijo poseer alto o muy alto rendimiento. De allí que el rendimiento escolar como motivación de la deserción podría tener significancia en el grupo que tenía bajo o muy bajo rendimiento ya que tal situación origina alejamiento de la escuela como lo expresan Vargas y Valadez (2016) y Tinto (2005).

Tabla 5

Rendimiento escolar de las adolescentes antes de su embarazo o actual

\begin{tabular}{c|c|c}
\hline $\begin{array}{c}\text { Rendimiento escolar antes del } \\
\text { embarazo o actual }\end{array}$ & Frecuencia & Porcentaje \\
\hline Muy bajo & 17 & 7,1 \\
Bajo & 98 & 41,8 \\
Alto & 108 & 46,1 \\
Muy alto & 12 & 4,9 \\
Total & 235 & 100,0 \\
\hline
\end{tabular}

Fuente: Elaboración propia. Datos obtenidos de una muestra de 235 adolescentes encuestadas en el Estado Táchira en el proyecto: El Táchira en Cifras 2014. Maternidad y embarazo en adolescentes (periodo de ejecución enero de 2014 - diciembre de 2017), en la Universidad Católica del Táchira, Venezuela.

Con quién vive actualmente la adolescente. Según los datos mostrados en la Tabla 6, un tercio de las madres adolescentes expresó que vive sin su pareja, con su familia de origen; más de un tercio con su pareja en casa de la familia de la pareja o de la familia propia y poco menos de un tercio en casa independiente con su pareja o sola. Por ende, este último grupo podría relacionarse con motivación de deserción escolar al vivir independientemente pues la madre posee más responsabilidades que cumplir tal como es planteado por Visu (2006 citado en Troncoso 2008); Espíndola y León (2002); Piña y Pontón (1997). 


\section{Tabla 6}

Con quién vive actualmente la adolescente.

\begin{tabular}{|c|c|c|}
\hline Con quién vive actualmente & Frecuencia & Porcentaje \\
\hline $\begin{array}{c}\text { Con tu pareja en casa de tu familia } \\
\text { de él }\end{array}$ & 45 & 19,2 \\
$\begin{array}{c}\text { Con tu pareja en casa de familia } \\
\text { de origen }\end{array}$ & 47 & 19,9 \\
\hline $\begin{array}{c}\text { Sin tu pareja en casa de tu familia } \\
\text { Con tu pareja en casa independiente }\end{array}$ & 78 & 33,3 \\
Con amigas(os) & 45 & 19,1 \\
Sola & 7 & 3,0 \\
\hline
\end{tabular}

Fuente: Elaboración propia. Datos obtenidos de una muestra de 235 adolescentes encuestadas en el Estado Táchira en el proyecto: El Táchira en Cifras 2014. Maternidad y embarazo en adolescentes (periodo de ejecución enero de 2014 - diciembre de 2017), en la Universidad Católica del Táchira, Venezuela.

\section{RESULTADOS DEL ESTUDIO REALIZADO EN EL NORTE DE SANTANDER}

Situación actual de estudio. En la investigación realizada, como se muestra en la Tabla 7, del total de adolescentes encuestadas, solo el 29,6 \% estudian, representando a 120 madres adolescentes; de allí que, para efectos del estudio de la deserción escolar, la cantidad de adolescentes de interés corresponde a 286.

\section{Tabla 7}

Situación actual de estudio.

\begin{tabular}{c|c|c}
\hline Actualmente estudia & Frecuencia & Porcentaje \\
\hline Sí & 120 & 29,6 \\
No & 284 & 70,0 \\
NS / NR & 2 & 5 \\
Total & 406 & 100,0 \\
\hline
\end{tabular}

Fuente: Elaboración propia. Datos obtenidos de una muestra de 406 adolescentes encuestadas en Norte de Santander en el proyecto: Maternidad adolescente en Norte de Santander (periodo de ejecución febrero 2015 - mayo 2016), en la Universidad Simón Bolivar sede Cúcuta.

\section{5}


La actividad principal antes del primer embarazo. Con respecto a las adolescentes que manifestaron abandono escolar, al indagar si antes estudiaban, como se visualiza en la Tabla 8, el 76,6 \% lo hacía antes del primer embarazo, bien fuese compartiendo con trabajo o solo estudiando. Es decir, que de las 286 adolescentes investigadas, 219 estudiaban antes del primer embarazo, con lo cual se denota el grave problema con respecto a la deserción escolar.

Tabla 8

Actividad principal antes del primer embarazo.

\begin{tabular}{c|c|c}
\hline Actividad principal & Frecuencia & Porcentaje \\
\hline Estudiar y trabajar & 17 & 5,9 \\
Solo estudiar & 202 & 70,7 \\
Solo trabajar & 59 & 20,6 \\
Ni estudiar ni trabajar & 8 & 2,8 \\
Total & 286 & 100,0 \\
\hline
\end{tabular}

Fuente: Elaboración propia. Datos obtenidos de una muestra de 406 adolescentes encuestadas en Norte de Santander en el proyecto: Maternidad adolescente en Norte de Santander (periodo de ejecución febrero 2015 - mayo 2016), en la Universidad Simón Bolivar sede Cúcuta.

\section{El abandono escolar antes y después del primer embarazo. Como se} muestra en la Tabla 9, de las adolescentes encuestadas que abandonaron los estudios, un poco menos de los dos tercios dejó de estudiar antes del primer embarazo y casi un tercio después del primer embarazo. Los resultados revelan que el 94,3\% de las adolescentes abandonaron estudios antes o después de su embarazo y $5,6 \%$ no sabe - no responde.

Tabla 9

Abandono escolar antes y después del primer embarazo.

\begin{tabular}{c|c|c}
\hline Abandono escolar & Frecuencia & Porcentaje válido \\
\hline Antes del primer embarazo & 180 & 62,9 \\
Después del primer embarazo & 89 & 31,1 \\
Después del segundo embarazo & 1 & 3 \\
NS / NR & 16 & 5,6 \\
Total & 235 & 100,0 \\
\hline
\end{tabular}

Fuente: Elaboración propia. Datos obtenidos de una muestra de 406 adolescentes encuestadas en Norte de Santander en el proyecto: Maternidad adolescente en Norte de Santander (periodo de ejecución febrero 2015 - mayo 2016), en la Universidad Simón Bolivar sede Cúcuta. 
Motivos del abandono escolar. Cuando se le preguntó al grupo de adolescentes que configuró la muestra (406), los motivos de dejar de estudiar antes del embarazo, se puede observar en la Tabla 10 que no quiso estudiar más, no hace falta en un 25,6\%, el haber quedado embarazada en un $24,6 \%$ o por lejanía de la escuela, necesitaba ganar dinero o no pasó los exámenes de entrada un $8,9 \%$, otros motivos como se casó, la familia necesitaba ayuda, tenía que cuidar los niños, enfermedad, dificultades de aprendizaje, oposición de los padres, violencia en la región, alcanzó el $40,9 \%$. La tabla revela que existe motivación del abandono escolar por haber quedado embarazada; sin embargo, otros factores son más influyentes, llegando a ser aproximadamente el $75 \%$.

Tabla 10

Motivos del abandono escolar antes del embarazo.

\begin{tabular}{|c|c|c}
\hline $\begin{array}{c}\text { Motivos del abandono escolar } \\
\text { antes del embarazo }\end{array}$ & Frecuencia & $\begin{array}{c}\text { Porcentaje } \\
\text { válido }\end{array}$ \\
\hline Quedó embarazada & 100 & 24,6 \\
\hline $\begin{array}{c}\text { No quiso estudiar más / Considera } \\
\text { que ya tiene suficiente estudio }\end{array}$ & 104 & 25,6 \\
$\begin{array}{c}\text { Institución educativa quedaba muy } \\
\text { lejos / Necesitaba ganar dinero / No } \\
\text { pasó exámenes de entrada }\end{array}$ & 36 & 8,9 \\
Otros motivos & 166 & 40,9 \\
Total & 406 & 100,0 \\
\hline
\end{tabular}

Fuente: Elaboración propia. Datos obtenidos de una muestra de 406 adolescentes encuestadas en Norte de Santander en el proyecto: Maternidad adolescente en Norte de Santander (periodo de ejecución febrero 2015 - mayo 2016), en la Universidad Simón Bolivar sede Cúcuta.

Como el 76,6 \% de las encuestadas (219) sí estudiaban o estudiaban y trabajaban antes del embarazo y $62,9 \%$ del total de encuestadas dijeron haber abandonado la escuela antes del embarazo (Tabla 9) que representan 180 madres, se conoció que las otras 39 se retiraron por razones que se aprecian en la Tabla 11. No querer estudiar representa el motivo en un poco más de la mitad en este grupo y, un tercio lo hizo por enfermedad; además se encontró la evidencia de otros motivos en un valor bajo como la lejanía de la escuela, necesidad de ganar dinero, oposición de los padres y de la pareja. 
Tabla 11.

Motivos del abandono escolar exceptuando el embarazo

\begin{tabular}{c|c|c}
\hline $\begin{array}{c}\text { Motivos del abandono escolar } \\
\text { después del embarazo }\end{array}$ & Frecuencia & $\begin{array}{c}\text { Porcentaje } \\
\text { válido }\end{array}$ \\
\hline No quiso estudiar & 21 & 53,8 \\
Enfermedad & 13 & 33,3 \\
\hline $\begin{array}{c}\text { Otros (el centro educativo quedaba } \\
\text { muy lejos, necesitaba ganar dinero, } \\
\text { oposición de los padres, oposición } \\
\text { de la pareja) }\end{array}$ & 5 & 12,9 \\
Total & 39 & 100,0 \\
\hline
\end{tabular}

Fuente: Elaboración propia. Datos obtenidos de una muestra de 406 adolescentes encuestadas en Norte de Santander en el proyecto: Maternidad adolescente en Norte de Santander (periodo de ejecución febrero 2015 - mayo 2016), en la Universidad Simón Bolivar sede Cúcuta.

\section{Rendimiento escolar de las adolescentes antes de abandonar sus estu-}

dios. En el grupo de adolescentes que abandonó los estudios, la indagación en cuanto a su rendimiento académico, dijo menos de la mitad manifestó que era bajo o muy bajo en contraposición al resto que dijo poseer alto o muy alto rendimiento (ver Tabla 12). Se deriva que el rendimiento escolar pudo haber influido en la deserción escolar en el grupo que manifestó poseer bajo o muy bajo su rendimiento en el sentido del desapego que trae como consecuencia ir mal en los estudios y que conduce a veces a la autoexclusión como lo expresa Troncoso (2008).

Tabla 12

Rendimiento escolar antes de abandonar los estudios.

\begin{tabular}{c|c|c}
\hline $\begin{array}{c}\text { Rendimiento escolar antes de } \\
\text { abandonar los estudios }\end{array}$ & Frecuencia & Porcentaje \\
\hline Muy bajo & 21 & 7,3 \\
Bajo & 106 & 37,1 \\
Alto & 135 & 47,2 \\
Muy alto & 19 & 6,6 \\
NS / NR & 5 & 1,7 \\
Total & 286 & 100,0 \\
\hline
\end{tabular}

Fuente: Elaboración propia. Datos obtenidos de una muestra de 406 adolescentes encuestadas en Norte de Santander en el proyecto: Maternidad adolescente en Norte de Santander (periodo de ejecución febrero 2015 - mayo 2016), en la Universidad Simón Bolivar sede Cúcuta. 
Con quién vive actualmente la adolescente. La Tabla 13 muestra que de las adolescentes que abandonaron la escuela, un poco más de la mitad vive sin su pareja con su familia de origen, menos de un tercio con su pareja en casa de la familia de él o con la suya propia, y un valor similar al anterior en casa independiente con su pareja o sola; es con respecto a este último grupo que podría derivarse algún motivo para la ocurrencia de la deserción escolar ante las obligaciones y compromisos adquiridos de acuerdo al planteamiento de Visu (2006, citado por Troncoso, 2008).

Tabla 7

Con quién vive actualmente la adolescente.

\begin{tabular}{|c|c|c|}
\hline Con quién vive actualmente & Frecuencia & Porcentaje \\
\hline Con tu pareja en casa de tu familia & 27 & $9,4 \%$ \\
\hline $\begin{array}{l}\text { Con tu pareja en casa de la familia } \\
\text { de él }\end{array}$ & 39 & $13,5 \%$ \\
\hline $\begin{array}{c}\text { Sin tu pareja en casa de familia } \\
\text { origen }\end{array}$ & 146 & $51,0 \%$ \\
\hline Con tu pareja en casa independiente & 55 & $19,2 \%$ \\
\hline Con amigas(os) & 7 & $2,5 \%$ \\
\hline Sola & 10 & $3,4 \%$ \\
\hline NS / NR & 2 & 1 \\
\hline Total & 286 & 100,0 \\
\hline
\end{tabular}

Fuente: Elaboración propia. Datos obtenidos de una muestra de 406 adolescentes encuestadas en Norte de Santander en el proyecto: Maternidad adolescente en Norte de Santander (periodo de ejecución febrero 2015 - mayo 2016), en la Universidad Simón Bolivar sede Cúcuta.

\section{DISCUSIÓN DE RESULTADOS}

En el estado Táchira, al relacionar las adolescentes que estudiaban antes del embarazo con las que siguen estudiando después del mismo existe un 52,8 \% ${ }^{6}$ de deserción; sin embargo, de tal porcentaje, 38,4 \% dejó los estudios después del embarazo y 61,6\% abandonó la escuela antes del embarazo. De este porcentaje se involucran diversas motivaciones como: terminó los estudios en $27,5 \%$, no quiso estudiar/no hace falta en un

6 (estudiaban antes: $23,1+61,5)-(31,8 \%$ continúan estudios).

\section{9}


$34,8 \%$, escuela o liceo lejos de casa en 5,8 \%, costo de útiles, transporte, gastos en un $7,2 \%$ y $24,6 \%$ otros motivos.

En el departamento Norte de Santander, de las 286 madres que no estudian al momento de la encuesta, 219 lo hacían antes (estudiaban o estudiaban y trabajaban antes del embarazo), considerando el total de madres de la muestra este valor representa el 53,94\%, que significa el porcentaje de la deserción escolar de madres adolescentes en el contexto de investigación. Además del total de la muestra se conoció que el 24,6 \% (100 madres del total) expresó que abandonó por el embarazo, un porcentaje muy cercano al $50 \%$ expresó motivos vinculados con otras razones (se casó, la familia necesitaba ayuda, tenía que cuidar los niños, enfermedad, dificultades de aprendizaje, oposición de los padres, violencia en la región) y un 25,6 \% por no querer estudiar o porque considera que ya tiene suficiente estudio.

Así, se refleja en los resultados para ambos contextos que, ante la existencia de factores internos o externos, el estudiante toma la decisión de abandonar voluntaria o forzadamente sus estudios tal y como plantearon Troncoso (2008) y López et al. (2012). El embarazo no es la principal causa de la deserción escolar en las madres adolescentes como lo expresan Mazuera y Arias (2017), en las madres adolescentes estudiadas más del $60 \%$ de ellas, en ambos contextos para el momento de la investigación, habían abandonado la escuela antes del primer embarazo.

Ante las respuestas ofrecidas por el grupo de encuestadas en el estado Táchira con respecto a su situación de vida, podría considerarse que al vivir independientemente con su pareja y vivir sola (que representa un resultado del 24,7 \% global), asumir el rol de madre, ama de casa y por ende llevar la vida de una adulta podría conducir al abandono en los estudios. En el departamento Norte de Santander, los resultados obtenidos con respecto a vivir de modo independiente, bien sea con su pareja o sola luego del embarazo, involucran un 22,6\% global, con el cual se puede considerar que 
la madre adolescente se dedicó a labores del hogar y cuidados del recién nacido. De este modo las ideas de Visu (2006, citado por Troncoso, 2008); Espíndola y León (2002); Piña y Pontón (1997), cobran valor ya que la realización de las tareas del hogar y el convertirse de adolescente a adulta, les hace decidir abandonar los estudios.

Con respecto al rendimiento académico bajo o muy bajo manifestado en un 48,9\% de las encuestadas en el estado Táchira, y de 44,4\% global para los mismos indicadores en las madres adolescentes estudiadas de Norte de Santander, se prevé una situación de desapego con la institución educativa y al no existir identidad con la escuela se presenta la toma de decisiones relacionada con el abandono, como lo plantean Vargas y Valadez (2016); Tinto (2005) y Troncoso (2008).

Por otra parte, se revela que en la deserción escolar ocurrida en las madres adolescentes estudiadas en el estado Táchira y en Norte de Santander, un factor que interviene es precisamente el embarazo, como lo han expresado Goicovic (2002) y Azevedo et al. (2013); no obstante se integra a otras razones, como no querer estudiar, enfermedad, el costo y otras motivaciones.

\section{CONCLUSIONES}

En la ocurrencia de la deserción escolar en las madres adolescentes se integran diversos factores como lo han expresado estudios de la Universidad Nacional de Colombia (2010), Abril et al. (2008), Visu (2006, citado por Troncoso, 2008); Corporación Colombia Digital (2012); Estrada (2014) y Woods (2002). Además, hay motivos en efectos posteriores al embarazo como vivir independientemente, bien sea sola o con la pareja, así como el rendimiento académico de la madre adolescente.

En la deserción escolar en madres adolescentes como fenómeno complejo, 
interviene el contexto social regional, como lo plantea Corporación Colombia Digital (2012). En los dos ámbitos que sirvieron para realizar los estudios se analizaron las mismas variables y se han obtenido regularidades que bien pueden ser empleadas para explicar la deserción escolar. De allí, que para acometer el problema de la deserción escolar en lo que respecta al embarazo y maternidad adolescente, las estrategias que pudiesen contribuir a su reducción y hasta eliminación, pueden construirse en conjunto o, al ser establecidas en alguno de los dos contextos podrían ser utilizadas por el otro, más aun, cuando en cada país se busca lograr los Objetivos de Desarrollo Sostenible (Organización de las Naciones Unidas, 2015).

\section{REFERENCIAS BIBLIOGRÁFICAS}

Abril, E., Román, R., Cubillas, M. J. y Moreno, I. (2008). ¿Deserción o autoexclusión? Un análisis de las causas de abandono escolar en estudiantes de educación media superior en Sonora, México. Revista Electrónica de Investigación Educativa, 10(1), 1-16. Recuperado de http:// redie.uabc.mx/vol10no1/contenido-abril.html

Aguilar-Mejía E, Viniegra-Velázquez, L. (1999). Deserción escolar y maltrato por parte de los profesores. Parte 1. Rev Med IMSS 37(4), 279-286. Recuperado de http://web.a.ebscohost.com/ehost/detail/ detail?vid=26ysid=c2ae8734-8509-43f6-b7ec-50461e097286\%40sessionmgr4010yhid=4204ybdata $=$ Jmxhbmc9ZXMmc210ZT1laG9zdC1saXZl\#AN=4236313ydb=a9h

Azevedo, J.P., Favara, M., Haddock, S. E., López-Calva, L.F., Müller, M. y Perova, E. (2013). Embarazo Adolescente y Oportunidades en América Latina y el Caribe. Informe sobre maternidad temprana, pobreza y logros económicos. Washington: Banco Mundial. Recuperado de http://documents.worldbank.org/curated/en/983641468238477531/pdf/831670WP0SPANI0Box0382076B00PUBLIC0.pdf

Bourdieu, P. y Passeron, J.C. (2002). La reproducción. Elementos para una teoría del sistema de enseñanza. Madrid, España: Editorial Popular.

Corporación Colombia Digital (2012). Deserción escolar. Encuesta Na- 
cional de Deserción Escolar. Bogotá: Colombia digital. Recuperado de https://colombiadigital.net/images/stories/simpade/desercion-escolar-encuesta.jpg

Departamento Administrativo Nacional de Estadísticas (DANE). (2016). Nacimientos 2016 Preliminar. Recuperado de https://www.dane.gov. co/index.php/estadisticas-por-tema/salud/nacimientos-y-defunciones/ nacimientos/nacimientos-2016

Espíndola, E. y León, A. (2002). La deserción escolar en América: Un tema prioritario para la agenda regional. Revista Iberoamericana de educación, (30). Recuperado de http://www.rieoei.org/rie30a02.htm

Espinoza Díaz, O., Castillo Guajardo, D., González, L. E., Loyola Campos, J., Santa Cruz Grau, E. (2014). Deserción escolar en Chile: un estudio de casos en relación con factores intraescolares. Educ., 17(1), 32-50. Recuperado de http://web.a.ebscohost.com/ehost/ detail/detail?vid=6ysid=c2ae8734-8509-43f6-b7ec-50461e0972 $86 \% 40$ sessionmgr4010yhid $=4204 \mathrm{ybdata}=$ Jmxhbmc9ZXMmc210ZT1laG9zdC1saXZl\#AN=96250135ydb=fua

Estrada, M. (2014). Afiliación juvenil y desafiliación institucional. El entramado complejo de la deserción en la educación media RMIE, 19(61), 431-453. Recuperado de http://web.a.ebscohost.com/ehost/pdfviewer/pdfviewer?sid=c2ae8734-8509-43f6-b7ec-50461e097286\%40 sessionmgr4010yvid $=37 \mathrm{yhid}=4204$

Fernández, L., Carro, E., Oses, D. y Pérez, J. (2004). Caracterización del recién nacido en una muestra de gestantes adolescentes. Rev Cubana Obstet Ginecol, 30(2). Recuperado de http://scielo.sld.cu/scielo. php?pid=S0138-600X2004000200003yscript $=$ sci_arttext

Fierro, J. (2010). Análisis estadístico univariado, bivariado y variables control. Recuperado de http://chitita.uta.cl/cursos/2012-1/0000104/recursos/r-25.pdf

Fondo de las Naciones Unidas para la Infancia (UNICEF). Venezuela (2017a). Panorama general. Recuperado de https://www.unicef.org/venezuela/spanish/overview_11420.htm 
Fondo de las Naciones Unidas para la Infancia (UNICEF). Venezuela (2017b). Indicadores básicos. Recuperado de https://www.unicef.org/ venezuela/spanish/overview_13275.htm

Fondo de Población de las Naciones Unidas (UNFPA) (2014). Boletín Informativo (140). Recuperado de http://venezuela.unfpa.org/doumentos/ BOLETIN\%20UNFPA\%20NRO\%20140\%202014.pdf

Fuenmayor D., Sulbarán, M. y Bargarza, Y. (2014a). Intervención educativa sobre prevención de embarazo en la adolescencia. Revista Médica Electrónica PortalesMedicos.com. 1-9. Recuperado de http://www.revista-portalesmedicos.com/revista-medica/ prevencion-embarazo-adolescencia/4/

Fuenmayor D., Sulbarán, M. y Bargarza, Y. (2014b). .Intervención educativa sobre prevención de embarazo en la adolescencia. Revista Médica Electrónica PortalesMedicos.com. 1-9. Recuperado de http://www.revista-portalesmedicos.com/revista-medica/ prevencion-embarazo-adolescencia/2/

Goicovic Donoso, I. (2002). Educación, deserción escolar e integración laboral juvenil. Última Década, 10(16), 11-52. Recuperado de http://www.scielo.cl/scielo.php?script=sci_arttextypi$\mathrm{d}=\mathrm{S} 0718$-22362002000100002ylng=esynrm=iso

Henao Escovar, J., González, C. y Vargas Trujillo, E. (2007). Fecundidad adolescente, género y desarrollo. Territorios, 16-17, 47-70. Recuperado de https://www.researchgate.net/profile/Elvia_Vargas_trujillo/ publication/28313173_Fecundidad_adolescente_genero_y_desarrollo_ Evidencias_de_la_investigacion/links/02bfe5126e7e27c3a3000000/ Fecundidad-adolescente-genero-y-desarrollo-Evidencias-de-la-investigacion.pdf

Hernández, R., Fernández, C. y Baptista, P. (2006). Metodología de la Investigación. (4a ed.). México: McGraw-Hill.

Hopkins J., Lambrou, N., Morse, A. y Wallach, E. (2005). Ginecología y Obstetricia. Madrid, España: Editorial Marbán. 
Issler J. R. (2001). Embarazo en la adolescencia. Revista de Posgrado de la Cátedra VIa Medicina, (107), 11-23. Recuperado de http://med.unne. edu.ar/revista/revista107/emb_adolescencia.html

López Gutiérrez, I., Marín Fontela, G. y García Rodríguez, M. E. (2012).

Deserción escolar en el primer año de la carrera de Medicina. Revista de Educación Médica Superior, 26(1), 45-52. Recuperado de http:// scieloprueba.sld.cu/pdf/ems/v26n1/ems05112.pdf

Magallanes Díaz, E. A. (2015). Condiciones socioeconómicas y educativas de las mujeres primíparas según Censo 2011 en el Estado Táchira. En Universidad Católica del Táchira. Observatorio Social del Estado Táchira. El Táchira en cifras 2014 Maternidad y embarazo en adolescentes. 65-73. San Cristóbal, Venezuela: LITHO ARTE C.A.

Magallanes Díaz, E. A., Albornoz Arias, N., Mazuera Arias, R., Machado M, J. E, Ramírez L, R. H., Vivas Franco, C. Z., Aleta A, J. D. (2015). El Táchira en cifras 2014. Maternidad y Embarazo en adolescentes. San Cristóbal, Venezuela: Observatorio Social, Universidad Católica del Táchira. Recuperado de: www.ucat.edu.ve/oset

Mazuera Arias, R. y Albornoz Arias, N. (2017). Maternidad adolescente, desigualdad social y exclusión educativa en el Norte de Santander (Colombia) y Táchira (Venezuela). Espacio Abierto Cuaderno Venezolano de Sociología, 1(26), 121-137.

Mazuera Arias, R., Albornoz Arias, N.C., Ramírez Martínez, C., Carreño Paredes, M. T., Peinado Contreras, Y. C., Morffe Peraza, M. A., Gallardo Pérez, H. J. (2017). Maternidad adolescente en el Norte de Santander. Maracaibo, Venezuela: Universidad del Zulia.

Méndez Flores, A. (2011). Menarquia, la primera menstruación. Recuperado de http://blog.ciencias-medicas.com/archives/tag/menarquia Molina, M. S., Ferrada, C.N., Pérez, R. V., Cid, L. S., Casanueva, V. y García, A. (2004). Embarazo en la adolescencia y su relación con la deserción escolar. Rev Méd Chile, 132(1), 65-70. Recuperado de http:// www.scielo.cl/pdf/rmc/v132n1/art10.pdf

\section{5}


Organización de las Naciones Unidas (ONU) (2015). Objetivos de desarrollo sostenible. Recuperado de http://www.un.org/sustainabledevelopment/ es/objetivos-de-desarrollo-sostenible/

Organización Mundial de la Salud (OMS) (2009). Boletín de la Organización Mundial de la Salud (OMS) Recopilación de artículos, 87, 405-484.

Pacheco-Sánchez, C. I. (2016). Embarazo en menores de quince años: los motivos y la redefinición del curso de vida. Salud pública de México, 58(1), 56-61. Recuperado de http://www.scielo.org.mx/pdf/spm/v58n1/ v58n1a12.pdf

Piña Osorio, J. M. y Pontón Ramos, C. B. (1997). La eficiencia terminal y su relación con la vida académica. El posgrado en sociología y ciencia política de la UNAM. Revista Mexicana de Investigación Educativa, 2(3), 66-177. Recuperado de http://www.redalyc.org/articulo. oa? id $=14000306$

Real Academia (2017). Desertar. Recuperado de http://dle.rae. es/?id=Cx3aK42

República de Colombia (2011). Ministerio de Educación Nacional. Encuesta Nacional de Deserción Escolar (ENDE. Encuentro Regional 2011. Recuperado de http://www.mineducacion.gov.co/1621/articles-293672_archivo_pdf_presentacion.pdf

Tinto, V. (2005). Student Retention: What Next? National Conference on Student Recruitment, Marketing, and Retention, Washington, D.C., July 27-30. Recuperado de http://hr.fhda.edu/_downloads/Student-Retention-What-Next_pdf

Troncoso Arcos, J. (2008). Escuela e inclusión. Aprendizajes del seminario latinoamericano "Prevención de la deserción escolar, reinserción e inclusión educativa". Ministerio del Interior, División de Seguridad Pública, Ministerio de Educación, Fondo de las Naciones Unidas para la Infancia, UNICEF Recuperado de http://www.unicef.cl/web/wp-content/uploads/doc_wp/Escuela_e\%20_inclusion.pdf

Universidad Nacional de Colombia (2010). Informe final. Recuperado de 
http://www.mineducacion.gov.co/1621/articles-293674_archivo_pdf_ institucional.pdf

Vargas, E. D. y Valadez, A. (2016). Calidad de la escuela, estatus económico y deserción escolar de los adolescentes mexicanos. Revista Electrónica de Investigación Educativa, 18(1), 82-97. Recuperado de http:// redie.uabc.mx/redie/article/view/713

Weller, J. (2007). La inserción laboral de los jóvenes: características, tensiones y desafíos. Revista de la CEPAL, (92), 61-82. Recuperado de http://biblioteca.hegoa.ehu.es/system/ebooks/16575/original/Insercion_laboral_de_los_jovenes.pdf

Williamson, N. (2013). Maternidad en la niñez: enfrentar el reto del embarazo en adolescentes. New York: Equipo Editorial de la UNFPA. Recuperado de http://cdn.20m.es/adj/2013/10/30/2478.pdf

Woods, E. G. (2002). Reducing the Dropout Rate. School Improvement Research Series. Close-Up, (17) Recuperado de http://educationnorthwest.org/sites/default/files/ReducingtheDropoutRate.pdf

Cómo citar este capítulo:

Cuberos, M.A., Vivas-García, M., Albornoz-Arias, N., Mazuera-Arias, R., y Carreño-Paredes, M.T. (2017). Deserción escolar en las madres adolescentes en el contexto fronterizo Norte de Santander y Táchira. En Albornoz-Arias, N., Mazuera-Arias, R., Espinosa-Castro, J.F. (Ed.), adolescencia: su relación con la familia, educación y sexualidad. Un enfoque transdisciplinario (pp.135-168). Barranquilla, Colombia: Ediciones Universidad Simón Bolívar.

\section{7}

